\title{
PRESSURE, TEMPERATURE AND HUMIDITY MONITORING SYSTEM USING THE ARTY PLATFORM
}

\author{
S.C. CONSTANTIN, G. PREDUSCA, E. DIACONU
}

Valahia University of Targoviste, Faculty of Electrical Engineering, Electronics, and Information Technology E-mail: gabriel.predusca@valahia.ro,

\begin{abstract}
The paper aims to present the usefulness of Field Programmable Gate Array - based systems, the elasticity of hardware design for reuse, and the use of hardware and software resources. The proposed measurement system is a starting point for the design of a weather station capable of interpreting the variation of the size of atmospheric pressure, temperature, and humidity, which could make local weather predictions.
\end{abstract}

Keywords: FPGA, Arty, Artix-7, Barometer, BMP180, HTU21D

\section{INTRODUCTION}

Determined by the development of new types of sophisticated programmable devices, the digital hardware design process has changed radically in recent years. Unlike previous generations in which board-level design included many SSI chips that contained classic gates, virtually any digital design produced today is made of high-density devices. This is true not only for control devices such as processors and memories, but also for logic circuits such as registers and decoders [1-2].

FPGA (Field Programmable Gate Array) is a programmable logic device introduced in 1985 by Xilinx. One of the main features that define it is that it can be programmed by the user immediately after the manufacturing process unlike other devices that have implemented this function at the time of manufacture [35].

An FPGA consists of an array of configurable logic blocks surrounded by programmable I/O blocks, connected with programmable links.

\section{Hardware design of the application using Vivado 2015.4}

The application is made using Vivado 2015.4 software (figure 1) [6-7].

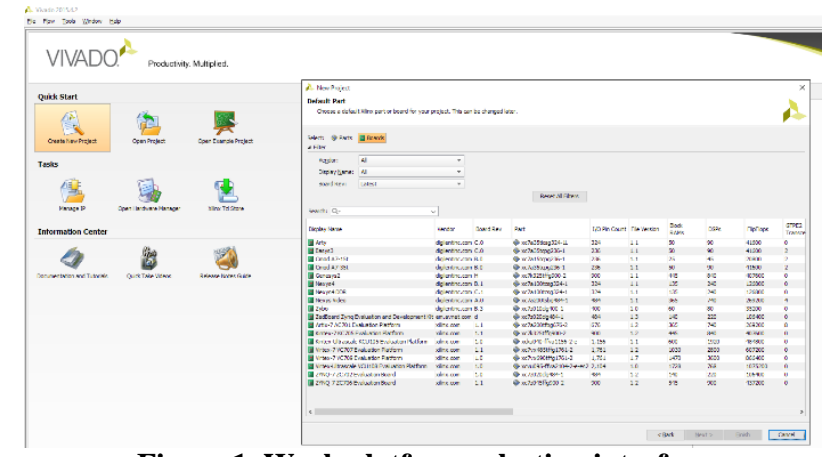

Figure 1. Work platform selection interface

\subsection{Barometer application}

To make this application we used: the Arty development platform, a pressure and temperature sensor from Bosch, BMP180, a standard liquid crystal display (LCD), with a line and 16 characters, which uses the HD44780 controller. The connection between the sensor and the platform was made through the $\mathrm{I} 2 \mathrm{C}$ serial communication interface, and the connection between the LCD and the platform was made through the GPIO ports (Figure 2).

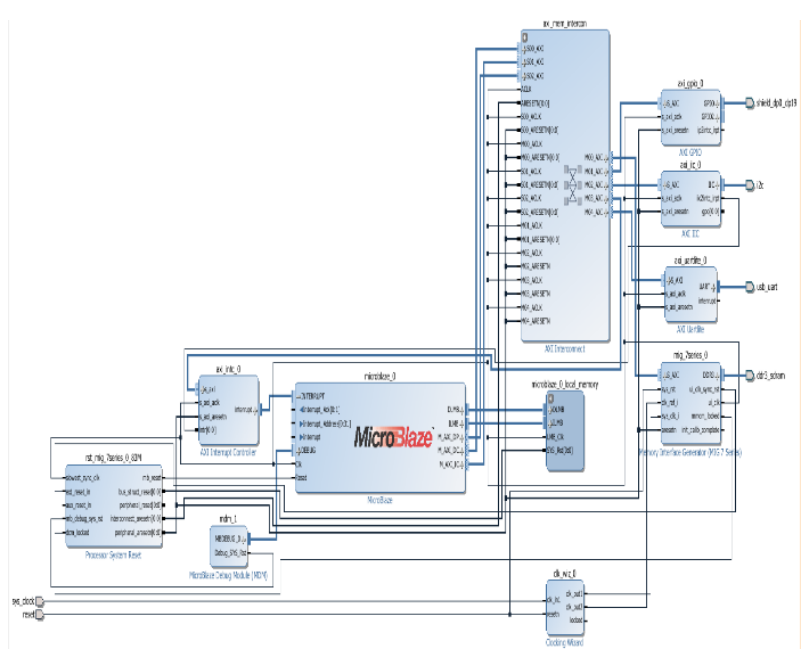

Figure 2. Final hardware diagram for barometer and hygrometer applications

The I2C standard is a multi-master communication standard that allows several devices to be connected in the same system via two communication lines: SDA data line and SCL clock line. The SCL line is always controlled by the master and the SDA can be controlled by both the master and the slave (figure 3 ). 


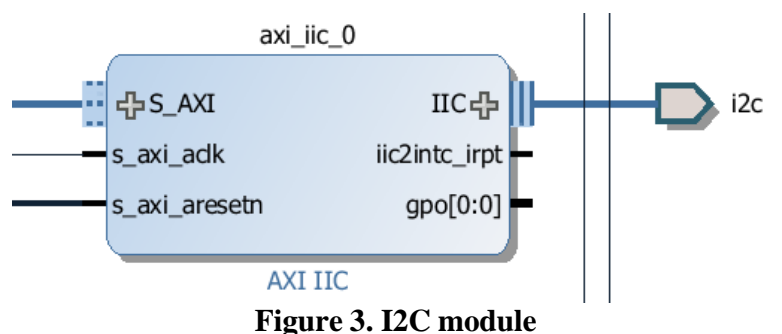

In Figure 2, if the I2C module controller generates a "transmission finished" interrupt it will be taken over by the interrupt controller and sent to the processor to execute the code structure (interrupt handler) that processes the end of the data transmission.

To control the LCD, we need a GPIO port (general purpose input output) and a $5 \mathrm{~V}$ voltage source, which are accessible on the Arty platform. The screen also needs a potentiometer to adjust its contrast. I connected it with the ends between GND and $+5 \mathrm{~V}$, and the cursor on pin 3. For this application I chose a $10 \mathrm{~K}$ potentiometer.

The LCD connection to the Arty platform is made with the "Shield pins 0 through 19" element. Vivado software will automatically connect this component to the AXI GPIO module (Figure 4).

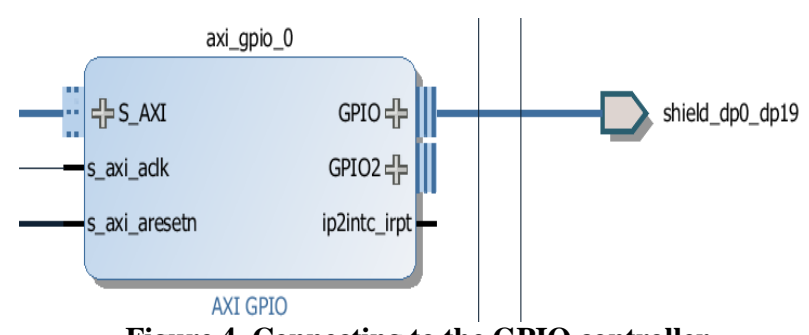

Figure 4. Connecting to the GPIO controller

The "AXI GPIO" controller can also be used to generate interrupts (for example if I had buttons connected).

For communication between the Arty platform and the PC, the USB interface is used, through which software debugging can be done. The element that does this is "USB UART" which has two elements "AXI Uartlite" and "usb_uart" (figure 5).

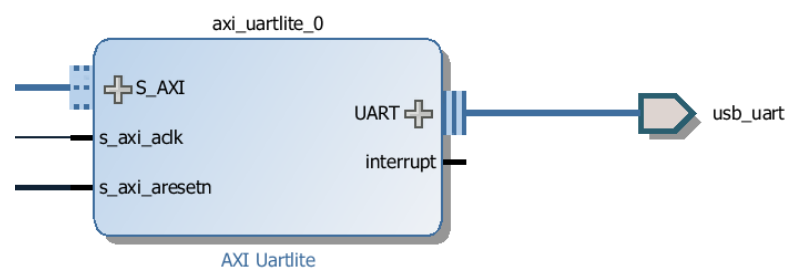

Figure 5. "AXI Uartlite" module and "usb_uart" interface

When generating the software project, two files are automatically generated, one with the extension "hw" and another with the extension "bsp". The bsp file extension is different in that it specifies new drivers for the I2c.c module, the interrupt module, and the UART module:
- uartlite_v3_a - contains functions and structures that allow communication with the outside (PC) on the serial interface.

- iic_v3_1 - contains functions and structures that allow communication with $\mathrm{I} 2 \mathrm{C}$ devices.

- intc_3v_4 - contains interrupt handlers for modules that need such a thing.

The logic diagram of the software for the barometer application is given in Figure 6.

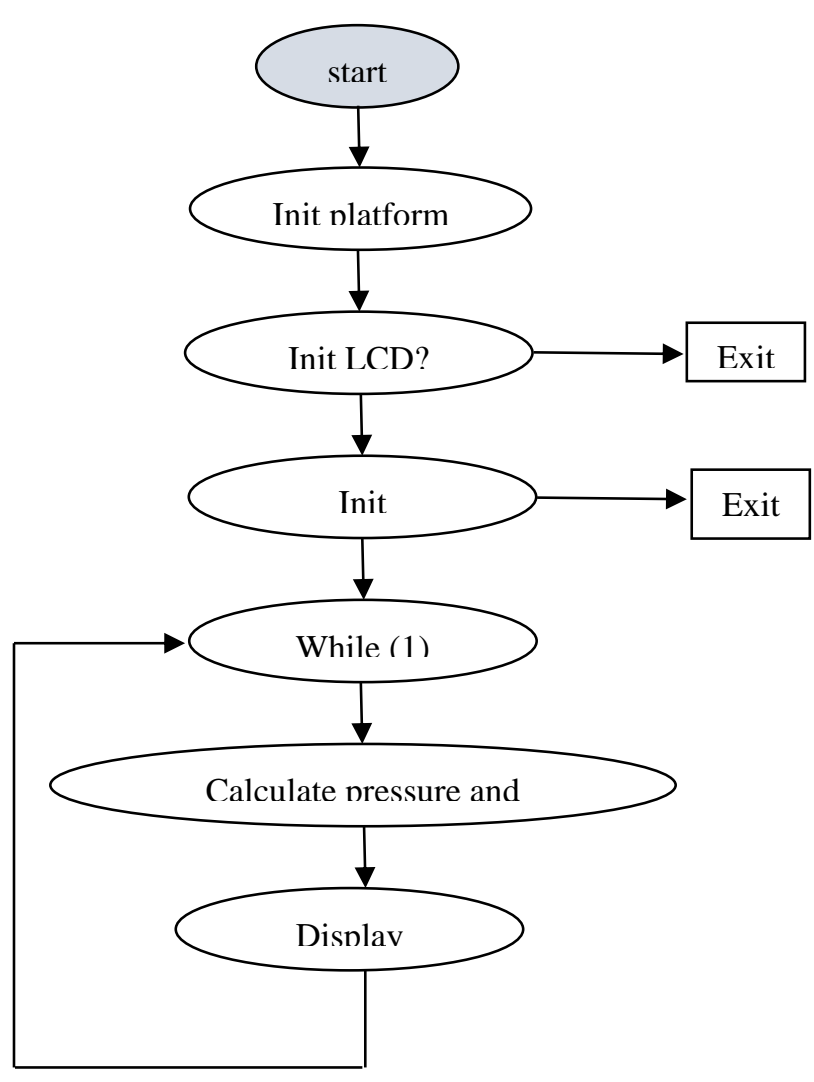

Figure 6. Logic diagram of the application

\subsection{Hygrometer application}

From the hardware and software point of view, the humidity measurement application is very similar to the pressure measurement application. The differences are in the name of the "Hygrometer_app" application and in the value reading driver. The "bmp180.c" driver has been replaced with "htu21d.c". The calculation methods are described in more detail in the software (figure 7). The logic diagram is the same as the one from "Barometer" with the difference that in the calculation process are accessed the calculation methods of humidity and temperature specific to the catalogue sheet of the sensor. 


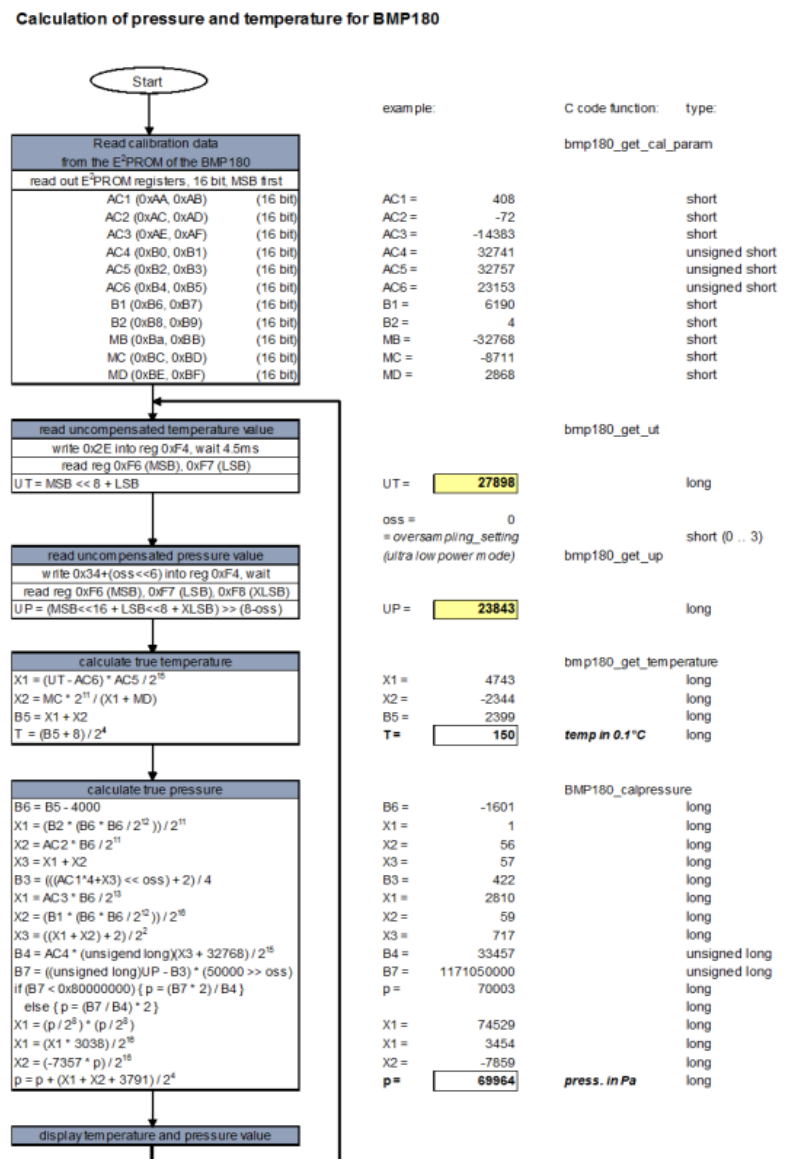

Figure 7. Pressure and temperature calculation formulas

\section{DESCRIPTION OF COMPONENTS AND DEVELOPMENT PLATFORM FPGA ARTY BOARD ARTIX-7}

\subsection{Artix-7 FPGA}

Arty is a ready-to-use development platform designed around Xilinx's Artix-7 FPGA (Figure 8).

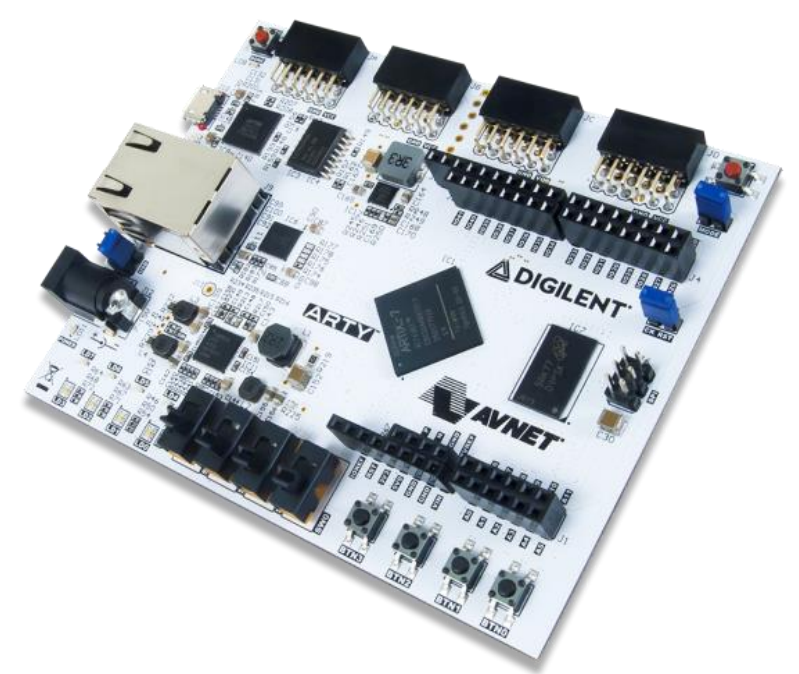

Figure 8. Arty development platform
Arty board features include:

- Xilinx Artix-35T FPGA.

- 33,280 logic cells in 5200 slices (each slice contains 6

LUT inputs and 8 flip-flops).

- 1,800 Kbit of fast RAM block.

- 5 zone of clock management, each with a closed phase loop (PLL).

- 90 DSP slices.

- Internal clock whose speed exceeds $450 \mathrm{MHz}$.

- analog-to-digital On-chip converter (XADC).

- Programmable JTAG and QUAD-SPI Flash.

- 256MB DDR3L with a 16-bit bus @ $667 \mathrm{MHz}$.

- 16MB Quas-SPI Flash [8-9].

\subsection{Barometric pressure sensor - BMP180}

The BMP180 (Figure 9) is the successor to the BMP085 representing a new generation of high-precision pressure sensors intended for consumer applications [10].

The BMP180's low power and low voltage electronics are optimized for use with mobile phones, GPS navigation devices or various outdoor equipment [11]. The BMP 180 offers superior performance with an I2C interface that allows easy system integration with a microcontroller. The BMP 180 also includes a temperature sensor.

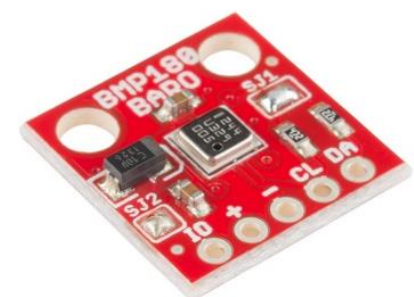

Figure 9. Barometric pressure sensor, BMP180

Technical specifications:

- It has an I2C interface.

- It has the supply voltage between the values: $1.8 \mathrm{~V}-3.8$ V.

- It has a low energy consumption.

- The sensor is calibrated at the factory.

- Small size.

- Has a temperature sensor included.

- Accuracy 300 to $1100 \mathrm{hPa}$.

- Measuring range: 300 to $1100 \mathrm{hPa}$.

\subsection{Humidity and temperature sensor, HTU21D}

The HTU21D digital humidity sensor is part of the category of dedicated sensors for humidity and temperature but also for OEM (Original Equipment Manufacturer) applications where reliable and accurate measurements are required. The direct interface with a microcontroller is possible with the humidity module and temperature outputs (Figure 10) [12]. 


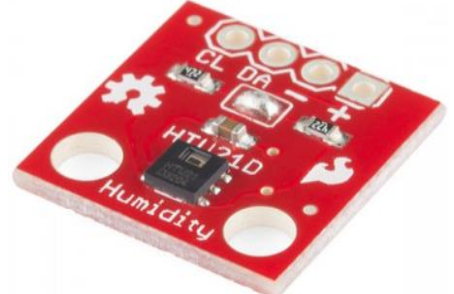

Figure 10. Humidity and temperature sensor, HTU21D

Each sensor is individually calibrated and tested. Low battery levels can be detected, and a checksum can improve communication reliability [13].

\section{Features:}

- Complete interchangeability, without calibration required under standard conditions.

- Individual marking for strict compliance with traceability requirements.

\subsection{Experimental results}

On the humidity side we can think of the simplest application. We all know how pretentious babies are. In the room where they live, it is good to ensure a constant humidity, although we do not need a precision instrument. The HTU21D sensor does just that. If we look in the catalogue sheet, the humidity calculation procedure is pressure dependent (Figure 11-15, Table 1).

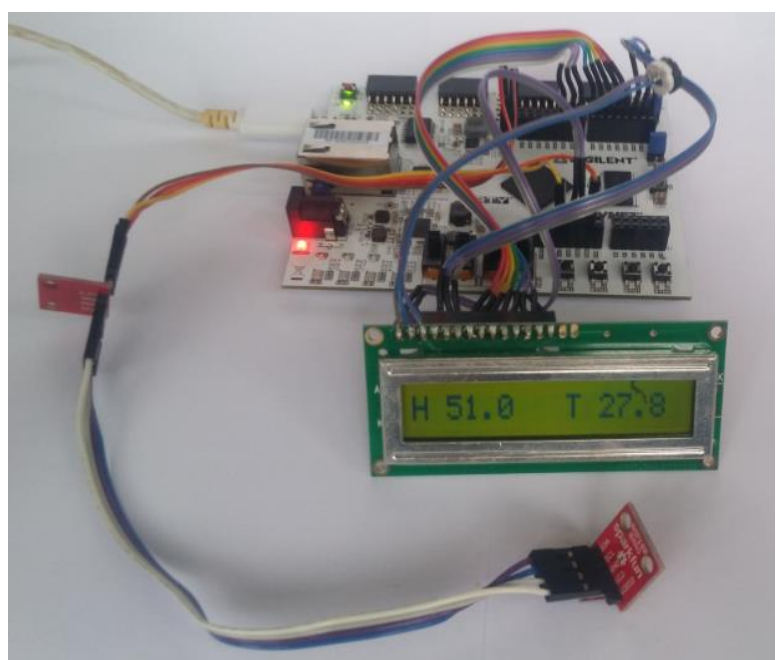

Figure 11. Hygrometer application result

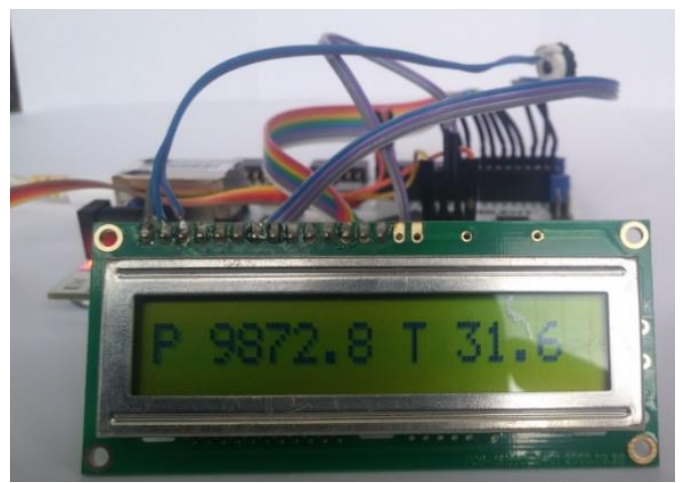

Figure 12. Barometer application result
Table 1. Variation of atmospheric pressure, humidity, and temperature over a period of 24 hours measured from hour to hour

\begin{tabular}{|c|c|c|c|}
\hline Time & $\begin{array}{c}\text { Pressure } \\
\text { (mBar) }\end{array}$ & $\begin{array}{c}\text { Humidity } \\
(\%)\end{array}$ & $\begin{array}{c}\text { Temperature } \\
\text { (C) }\end{array}$ \\
\hline 0:00 & 9989.30 & 46.00 & 19.10 \\
\hline 1:00 & 9980.70 & 46.00 & 18.80 \\
\hline 2:00 & 9976.10 & 47.00 & 18.70 \\
\hline 3:00 & 9975.00 & 48.00 & 18.50 \\
\hline 4:00 & 9972.10 & 48.00 & 18.30 \\
\hline 5:00 & 9971.60 & 49.00 & 18.30 \\
\hline 6:00 & 9965.20 & 48.00 & 18.70 \\
\hline 7:00 & 9975.20 & 47.00 & 18.90 \\
\hline 8:00 & 9986.10 & 46.00 & 19.10 \\
\hline 9:00 & 9991.10 & 45.00 & 19.30 \\
\hline 10:00 & 9996.80 & 45.00 & 20.20 \\
\hline 11:00 & 10002.40 & 44.00 & 21.40 \\
\hline 12:00 & 10006.50 & 44.00 & 22.50 \\
\hline 13:00 & 10006.70 & 43.00 & 23.30 \\
\hline 14:00 & 10007.10 & 44.00 & 24.50 \\
\hline 15:00 & 10007.40 & 45.00 & 25.60 \\
\hline $16: 00$ & 10008.10 & 46.00 & 26.10 \\
\hline 17:00 & 10007.70 & 46.00 & 26.20 \\
\hline 18:00 & 10007.60 & 46.00 & 25.70 \\
\hline 19:00 & 10007.20 & 45.00 & 25.40 \\
\hline 20:00 & 10006.40 & 47.00 & 24.30 \\
\hline 21:00 & 10006.50 & 48.00 & 22.70 \\
\hline 22:00 & 10005.90 & 48.00 & 21.30 \\
\hline 23:00 & 10005.10 & 47.00 & 20.30 \\
\hline
\end{tabular}

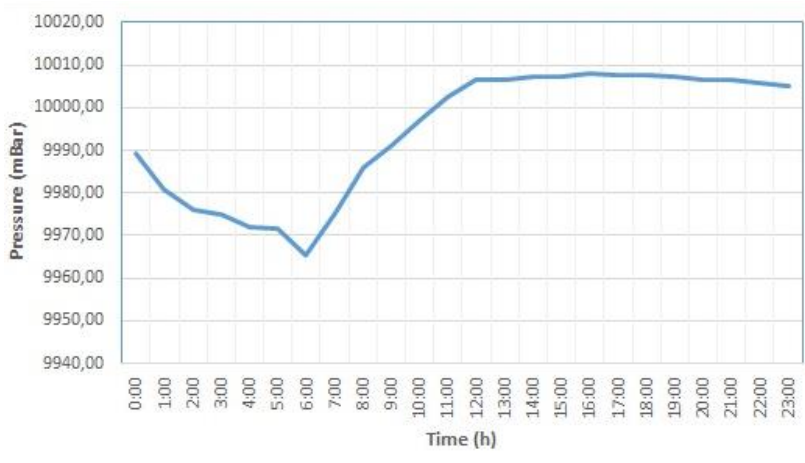

Figure 13. Graph of variation of atmospheric pressure over time 


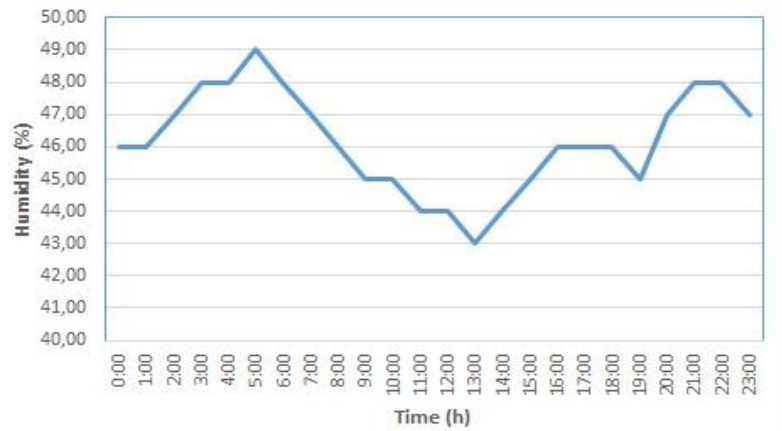

Figure 14. Graph of humidity variation over time over a 24hour period

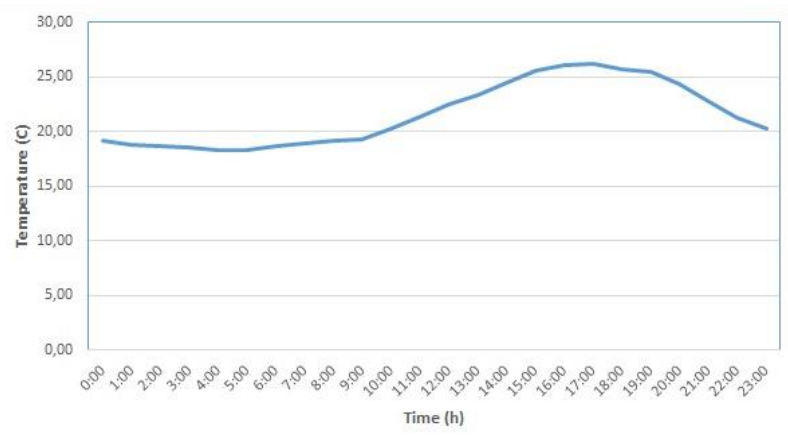

Figure 15. Graph of temperature variation over time

\section{RESULTS AND CONCLUSIONS}

The FPGA platform used is a very flexible platform. Although compared to dedicated microcontroller platforms, FPGAs are much more expensive financially, the spectrum of applications we can achieve is much larger.

The XILINX development environment of which the Vivado program is part as well as the Xilinx SDK are very well developed platforms. The advantage of the Xilinx SDK is that it generates what is called a "Board Support Package", a very easy-to-use "low level" driver library, which greatly simplifies the software development part.

As a conclusion, from the experimental determinations, we found that there are certain measurement differences between the temperature sensors incorporated on the 2 plates. These differences are mainly due to different construction methods. These sensors are rather experimental sensors for a learning platform. We found that the $\mathrm{I} 2 \mathrm{C}$ protocol is a simple to use protocol and is also convenient for hobby applications. The designed measuring devices that have been used in this paper cannot be used as accurate measuring instruments in any environment. For example, the barometer cannot be used to calculate altitude on aircraft. Instead, it can be used as a reference tool for weather stations to make weather forecasts in relatively short periods of time.

After experimenting with this procedure, I can tell you that compared to a commercial barometer the maximum error was $1 \mathrm{mBar}$ which is a success.
Also, the thermometer can be used in a greenhouse where we want to have a constant temperature and with the help of sensors, we can create a self-regulating environment in which the temperature can be set to certain values and kept constant.

With the help of the hygrometer, we can also create automated systems that keep certain rooms at constant humidity, which is used both in homes and in agriculture where certain greenhouse crops need a certain level of humidity (mushrooms). They can also be used in incubators, saunas, and museums. A hygrometer is also very useful in homes where it is necessary to ensure a constant humidity, especially in rooms where there are small children or people suffering from certain lung diseases.

\section{REFERENCES}

[1] Florian Ion, Electronică digitală - memorii ROM, RAM, circuite CPLD, FPGA, Editura Bibliotheca, Târgoviște, 2009.

[2] Florian Ion, Gabriel Predusca, Analiza si sinteza circuitelor numerice. Aplicații si probleme, Editura Bibliotheca, Târgoviște, 2006.

[3] Wael M. El-Medany, FPGA implementation for humidity and temperature remote sensing system, 14th International mixed-signals, sensors and systems test workshop (IMS3TW), 2008.

[4] Adel Mellit, H. Mekki, A. Messai, Soteris A. Kalogirou, FPGA - based implementation of intelligent predictor for global solar irradiation, part I: theory and simulation, Expert systems with applications 38, Elsevier, pp. 2668-2685, 2011.

[5] K. Sindhu, Y. Sri chakrapani, M. Kamaraju, FPGA implementation of irrigation control system, International Journal of Scientific \& Engineering Research, volume 5, issue 12, pp.1361-1364, December 2014.

[6] http://www.xilinx.com/support/download.html;

[7] http://www.xilinx.com/support/answers/66830.html;

[8] https://www.xilinx.com/products/boards-andkits/arty.html;

[9] https://digilent.com/reference/programmablelogic/arty/reference-manual?redirect $=1$;

[10] https://learn.sparkfun.com/tutorials/bmp180barometric-pressure-sensor-hookup-/all;

[11] Hao Xia, Xiaogang Wang, Yanyou Qiao, Jun Jian, Yuanfei Chang, Using multiple barometers to detect the floor location of smart phones with built-in barometric sensors for indoor positioning, Journal Sensor, Volume 15, pp.7857-7877, 2015.

[12] https://learn.sparkfun.com/tutorials/htu21dhumidity-sensor-hookup-guide;

[13] Zhuofu Liu, Jianwei Li, Meimei Liu, Vincenzo Cascioli, Peter W. McCarthy, In-depth investigation into the transient humidity response at the body-seat interface on initial contact using a dual temperature and humidity sensor, Journal Sensor, Volume 19, Issue 6, pp.1-16, 2019. 